\title{
Applying Pulse Width Modulation in Body Coupled Communication
}

\author{
Miltiadis Moralis-Pegios, Pelagia Alexandridou, and Christos Koukourlis \\ Telecommunications Systems Laboratory, Electrical and Computer Engineering Department, Democritus University of Thrace, \\ 67100 Xanthi, Greece \\ Correspondence should be addressed to Christos Koukourlis; c.koukourlis@gmail.com
}

Received 6 May 2015; Accepted 1 September 2015

Academic Editor: Zhenjiang Zhang

Copyright (C) 2015 Miltiadis Moralis-Pegios et al. This is an open access article distributed under the Creative Commons Attribution License, which permits unrestricted use, distribution, and reproduction in any medium, provided the original work is properly cited.

We study the application of Pulse Width Modulation (PWM) technique in Body Coupled Communication. The term Body Coupled Communication is used in order to specify that the human body or a part of it, such as an arm, is used as a path that transfers digital information. The digital information is either generated due to coupling of the body, for example, in medical equipment as a result of a measurement, or generated by external circuitry attached somewhere onto the body and is transferred to a terminal by touching it. In this paper, the latter case will be described, where, for illustration purposes, the touch of the human hand on a doorknob triggers the unlocking mechanism.

\section{Introduction}

As more mobile devices such as cellular phones, PDAs, mp3 players, tablets, and notebooks become necessary and almost indispensable nowadays, there is an increasing necessity for an easier and more comfortable method to connect each other. Body Area Networks (BANs) propose new ways of wireless communications that allow an interaction between the user and devices placed in close proximity or coupled directly with the user's body. There are several proposed technologies appropriate for a BAN. One of the candidate technologies is Body Coupled Communication (BCC) or Intrabody Communication (IBC) which was firstly proposed by Zimmerman [1]. Body Coupled Communication, in which the human body is used as a signal transmission medium, is becoming a promising solution for BANs including a large variety of interesting potential applications.

As signals pass through the human body, electromagnetic noise and interference have reduced influence on transmissions. This technology has several advantages over the conventional RF approaches. Since its operation is based on near-field coupling (galvanic or capacitive), most of the signal from the transmitter is restricted to the body area without the possibility to be collected or interfere with other external
RF devices. Moreover, since the communication frequency can be lowered without affecting any antenna size, the power consumption of the transmitter and the receiver is also much reduced compared to Bluetooth or Zigbee applications. According to BCC approach, a low frequency carrier (less than $1 \mathrm{MHz}$ ) is used; so no energy is propagated, minimizing remote eavesdropping and interference with other BANs. All the above characteristics make this technology a very promising solution for BANs, health monitoring applications, and identification systems like entering a security PIN in a bank automated teller machine terminal.

In this paper, a wearable identification system that uses BCC technology is proposed but mainly the application of a specific digital modulation technique in Body Coupled Communications is studied. This technique is a digital version of the well-known Pulse Width Modulation (PWM), which provides special spectral characteristics and simplicity of implementation. Generally speaking, PWM is an "analog" modulation technique which employs a square wave carrier. This modulation carries the information in the duty cycle of the modulated square wave carrier. In our case, we employ this digital version of PWM, named B-PWM, by using only two distinct duty cycles, as the two different symbols that represent the two logic states, one and zero. It will be 
explained later that the two distinct duty cycles are preferable to be complementary; that is, they must sum up to $100 \%$. For the case described later, we selected $25 \%$ and $75 \%$, representing "logic zero" and "logic one," respectively.

In order to have a very compact and small sized transmitter, we developed our modulator around a Microchip general purpose microcontroller, almost without any other component. It includes the clock oscillator and can be programmed in order to accomplish the modulation and also store the required identification data. It can be powered by a single 3 to $5.5 \mathrm{~V}$ power supply, which can be accommodated by one or two Lithium battery cells; or it can be charged by inductive coupling when in close proximity to the receiver, just before the transmission.

It is well known from the literature $[1,2]$ that a signal can be transferred via the human body by utilizing either of two methods: the galvanic method and the capacitive method. The specific digital modulation proposed in this paper is suitable for either case. Also, from the literature it comes out that most of the systems consider as appropriate for signal transmission through the human body a center frequency in the range of hundreds of $\mathrm{kHz}$ up to a few $\mathrm{MHz}$, for example, $500 \mathrm{kHz}$ [3]. Due to the body behavior, there is no guarantee that the receiver's frequency characteristic is flat. Instead, it depends on the electronics and the behavior of the human body itself. In an attempt to explain this behavior, some approaches $[1,4]$ propose an electrical model of the human body for BCC.

\section{Body Coupled Communication}

2.1. Propagation Methods. As referred above, two coupling methods have been proposed in the literature: the capacitive and the galvanic coupling. In both approaches, a pair of electrodes is used at both transmitter and receiver ends in order to carry out communication. Capacitive coupling makes use of a single "signal" electrode, while the second "ground" electrode remains on air. On the other hand, galvanic coupling requires direct contact between the pair of electrodes and the human body. A significant difference between these two approaches is that in the capacitive coupling the environment can affect the behavior and the quality of the transmitted signal, while in galvanic coupling approach communication is affected more by the body tissue dielectric parameters [5].

2.2. Modulation Methods. In previous studies, several modulation schemes have been tested. OOK [6] and FSK $[7,8]$ are the most commonly used modulation methods regarding Body Coupled Communication systems. In other studies [3, 9], MSK and BPSK are proposed. In our experiments, OOK, FSK, and PWM have been tested. Although OOK has low energy and spectrum requirements and the implementation is simple, it shows low tolerance to noise. On the other hand, although FSK overcomes noise effects to some extent, comparing to OOK and B-PWM, the implementation is complex and less energy efficient. The digital Pulse Width Modulation scheme proposed in this paper proves to be a suitable and appealing modulation technique for BCC technology, since it meets the basic requirements, that is, low energy consumption, simple implementation, and noise tolerance, as well as other practical advantages that will be described in Section 3.

2.3. Characterization. Before designing and implementing a WBAN (Wireless Body Area Network), one must consider the key design requirements of it. The present study is examined with the following criteria.

Suitability. The signal that passes through the human body must be suitable and not harmful for the human's health. In our implementation, carrier frequency ranges between $500 \mathrm{kHz}$ and $1 \mathrm{MHz}$ and the current flow is below $20 \mathrm{~mA}$; thus, it meets this requirement according to the international regulations $[10,11]$.

Reliability. Reliability is a crucial factor for every communication system. For that reason, we take into account the influence of the human body and external noise on the signal. The proposed method's spectral behavior is identical for both logic stages of the transmitted signal.

Energy Efficiency. There is no doubt that power consumption is of key importance in wearable technology. Thus, we have chosen just a low-power microcontroller for the transmitter's circuit.

Size. The device size is also a crucial issue for every wearable device. In the present implementation, the transmitter consists of a single low-power 8-pin microcontroller (that includes an internal oscillator) and one pair of electrodes.

Security. In this paper, due to the design of an identification system, security is a significant factor, although in the literature more sophisticated methods have been proposed [12]. The communication system presented here provides communication between two devices that both have direct contact to the body. The signal is transmitted only through the human body; that is, it is not radiated; so there is no possibility of eavesdropping or interference with other WBANs without the user's notice.

Data Rate. A high data rate is a challenge for every communication system. In the literature, data rates up to $10 \mathrm{Mbps}$ [13] have been mentioned. The nature of the application that is developed here does not require high data rate; so we merely set it to $10 \mathrm{kbps}$.

\section{Binary Pulse Width Modulation (B-PWM)}

3.1. Spectral Properties. The adoption of B-PWM is based on its inherent characteristic, as shown later, of occupying the same spectrum for both cases of symbol transmission (logic zero and logic one) due to the complementary values of selected duty cycles. This property is crucial for the front end stage of Body Coupled or Intrabody Communication designs 


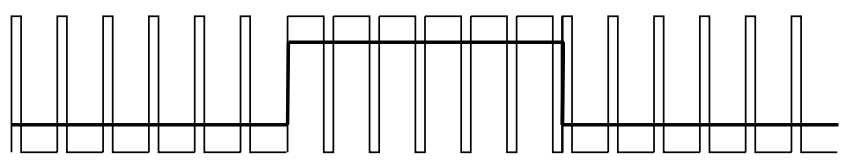

Figure 1: Pulse stream with two complementary duty cycles $(25 \%$ and 75\%) corresponding to logic " 0 " and logic " 1 ." The bold line on top is the associated average value with voltage levels corresponding to the logic states.

$[14,15]$, because the behavior of the body in association with the front end electronics usually exhibits strange or not easily predictable behavior.

The chopping action of B-PWM can move the spectrum of the modulated signal anywhere by changing the repetition rate of the carrier pulse stream. In Figure 1, two discrete pulse streams (symbols) of complementary duty cycles $(25 \%$ and $75 \%)$ are shown. We will show that the spectral content of all the segments (symbols) of the waveform is the same, under the assumption that the waveforms are periodic and they can be analyzed in Fourier series. The amplitudes of the corresponding harmonics prove to be equal but of $180^{\circ}$ phase difference.

It is well known that average value of a periodic waveform is as follows:

$$
A_{0}=A \cdot \frac{\tau}{T_{0}}
$$

where $A$ is the amplitude of the pulses, $\tau$ is the "on" time, and $T_{0}$ is the period of the waveform while the ratio $\tau / T_{0}$ is the duty cycle. If we consider the two distinct symbols, $s_{0}$ and $s_{1}$, of complementary duty cycles, it is obvious that $s_{1}$ can be produced from $s_{0}$ with a simple linear process:

$$
s_{1}(t)=-s_{0}(t)+A
$$

that is, $s_{1}$ is derived from $s_{0}$ when inverted and raised by $A$ volts plus a time shift. Since the time shift does not generate any new spectral components and because of this linear relation, the spectral lines of both waveforms are identical, one by one, despite being with phase shift of $180^{\circ}$ (i.e., inverted), with the only exception among the spectra of the two waveforms to be the spectral line at zero Hertz, that is, at DC. This similarity in spectral content of the two symbols employed ensures that the behavior of the body/receiver combination will be identical for both cases of signaling, that is, logic "one" and logic "zero."

As mentioned earlier, in our case, the B-PWM modulated carrier is generated at the output of a microcontroller powered by a single supply. This means that the transmitted waveform has a certain DC content during the duration of one bit which is equal to the mean value of the waveform. This DC content (which differs for the two logic levels) is where the digital information is stored. The whole system behaves as high pass stage not only due to the coupling with the body but, in some cases, due to the front end of the receiver as well. So, in any case, someone can consider that the information, which lies in the DC component of each symbol, would be lost due to this high pass behavior. Actually, as it will be shown later, the DC component is indeed lost, but not the information, since the information lies in the zerocrossings of the modulated waveform and a restoration of the received waveform in rail-to-rail voltage levels takes place at the receiver. Since the duty cycle percentages stay at the prescribed values, there is no loss of information, as long as the demodulator is a simple Low Pass Filter.

3.2. Optimal Duty Cycle Selection. According to Figure 1, we can initially assume that the difference between the two complementary values of duty cycle should be the maximum in order to have the most distinct levels of the two mean values (bold line in Figure 1). This drives to an extreme selection of $0 \%$ and $100 \%$ duty cycle pair, which is not acceptable because there is no chopping of the signal as referred in Section 3.1. On the other hand, the maximization of the voltage difference between the two levels at the demodulation stage, as described above, is not the only criterion. This will be clarified later.

In Figure 2, a part of a modulated waveform, as it should appear after the front end of the receiver, is shown. Due to the coupling and the electronics of the receiver, the waveform is weighted above and below a horizontal straight line in order to result in zero average value. As described earlier, due to the capacitive coupling of the receiver/detector and the high pass behavior of the front end, the low frequency content of the transmitted signal (the mean value included) will be lost. In Figure 3, the output of the transmitter is shown while in Figure 4 the block diagram of the receiver is given. The actual views of the signals (oscilloscope captures) at the receiver are the ones shown in Figure 5 after the 1st and the 2nd stages. In the upper trace of Figure 5, an oscilloscope capture at the output of the first (current to voltage converter) stage is shown. It is clear that the low frequency content is lost and the waveform is weighted above and below the zero mean value (zero volts line). In the lower trace of the same figure, the output of the second (inverting voltage comparator) stage is given and it is shown that the data carrying waveform is restored in to its two original levels. Also in Figure 6 (upper trace), the same restored waveform at the voltage comparator's output is given in different time scale, followed (lower trace of Figure 6) by the waveform produced at the output of the demodulating Low Pass Filter. In the lower trace of Figure 6, it is shown that the two different duty cycles give two distinct voltage levels despite being rounded, which correspond to the two logic states of the data. The same waveform (output of the LPF) is also shown at the upper trace of Figure 7 in order to be compared to the output of the Pulse Shaping Stage of the receiver.

As stated earlier, at first glance, in Figure 5 (upper trace), it seems that the information lying in the voltage levels of Figure 1 (bold line) is lost. Fortunately, this is not the case, because the information actually lies in the zero-crossings of the modulated signal, according to the duty cycle: the signal, as shown in Figure 2 or Figure 5 (upper trace), will be restored at the original levels at the receiver after a voltage comparator stage. Since the signal can be distorted and noise is added, it is 


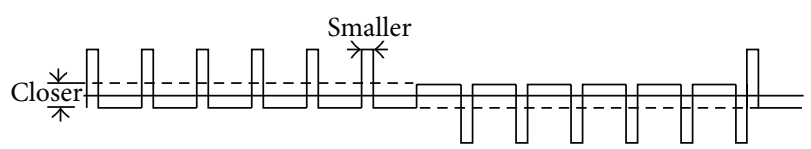

(a)

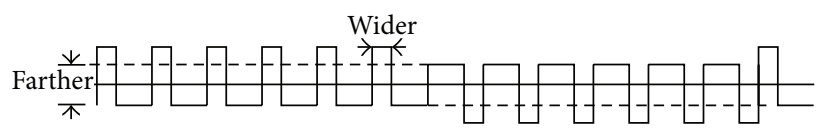

(b)

FIGURE 2: Two different cases of complementary duty cycles are shown. The left trace (a) has more distinct duty cycles. The right trace (b) has duty cycles closer to $50 \%$. Both traces are weighted above and below the zero mean value (horizontal) line.

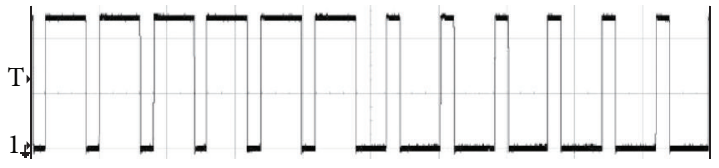

FIGURE 3: Oscilloscope capture of the B-PWM waveform at the output of the transmitter, similar to the one of Figure 1.

now evident that the two levels (corresponding mean values or envelopes) of the signal close to the zero line, Figure 2, must be as distinct as possible. This requirement drives at duty cycles closer to $50 \%$. This is opposite to our former aspect of the most distinct duty cycles. In Figure 2, the two cases of modulated waveforms of the same initial amplitude but of different pairs of complementary duty cycles are shown. The left trace (a) has more distinct duty cycles, more apart from $50 \%$, while the right trace (b) is closer to $50 \%$. It is evident that the right trace (b) has an advantage since the two envelopes around the straight (zero mean value) line are in larger distance (more distinct) than the corresponding ones of the left trace (a). As the $3 \mathrm{rd}$ stage, the demodulating one, is a Low Pass Filter which accepts the restored but still modulated B-PWM waveform and has to give the demodulated data at its output, the most distinct the duty cycles are, the best the voltage level separation appears. So, a compromise at the duty cycle selection is necessary. Since the lower value of the duty cycle has to be somewhere among $0 \%$ and $50 \%$ while the upper value of the duty cycle has to be in the range among $100 \%$ and $50 \%$, the average values of the two ranges are selected, that is, $25 \%$ and $75 \%$, in order to maintain the complementarity of the pair (summing $100 \%$ ), because in this way we obtain the most distinct voltage levels at the output of the demodulation filter while preserving the chopping action of the transmitted waveform.

\section{Hardware Implementation and Experimental Results}

In order to evaluate the proposed modulation, a prototype transmitter-receiver pair has been built. As referred above, the whole transmitter, including the B-PWM modulation, is implemented around a Microchip general purpose microcontroller which also includes the clock oscillator. The microcontroller generates a 64-bit key at an output pin. The PWM signal is applied to the human body via a coupler. In the present implementation, the coupler is composed of two copper electrodes separated by a $5 \times 2.5 \mathrm{~cm}$ dielectric
(Figure 8). The one electrode is connected to the ground, while the second one is connected to the output pin.

The coupler's position on the human body influences the communication too $[16,17]$. In our experiments, the pair of the transmitter's electrodes was placed along the hand. It turned out that the signal's strength is higher when applied on the forearm and lower when applied over the bones of the wrist. Also, the signal's strength varies in relation to the carrier frequency too, as it is shown in Figure 9.

In Figure 10, the experimental setup is shown. The battery-powered transmitter (small board) is placed on a human hand. The electrode is held in place, touching the skin, by the assistance of two rubber bands.

On the receiver's side, there is a single electrode that receives the B-PWM signal by simply touching it. Various types of electrodes were tested for the receiver. It turned out that a single small sized electrode was sufficient. The detected B-PWM signal, after being restored, passes through a simple Low Pass Filter with a cutoff frequency above the data rate in order to get the average value and thus demodulate it. The demodulated signal is restored in the appropriate form for the USART peripheral and enters the microcontroller. The microcontroller compares the input data with the valid key pool that has already been in memory. If the input data matches to a valid key, an electrically operated lock opens. If they do not match or there is no signal, the lock remains closed and a relevant message is shown at the LCD screen. A similar mechanism could be adopted and applied to bank ATM systems, providing additional security to the identification of the user.

\section{Conclusion}

In this paper, we presented B-PWM as a modulation scheme suitable for Body Coupled Communication. B-PWM proved to be a promising modulation technique for such an application. An identification system that uses BCC was developed and tested. The transmitter and the receiver are isolated and communicate to each other only through the human body.

The major advantage of the proposed method is its simplicity of implementation, especially at the modulator, comparing to [18], for example. This is most critical when the Body Coupled Communication is to be applied in general purpose devices including wearable electronic devices applications like wrist-type computers such as smartwatches. The proposed modulation can be easily implemented almost only in software of any microcontroller based system without the need of any other component, except a contact surface to the skin. Also, the method can be extended in higher 


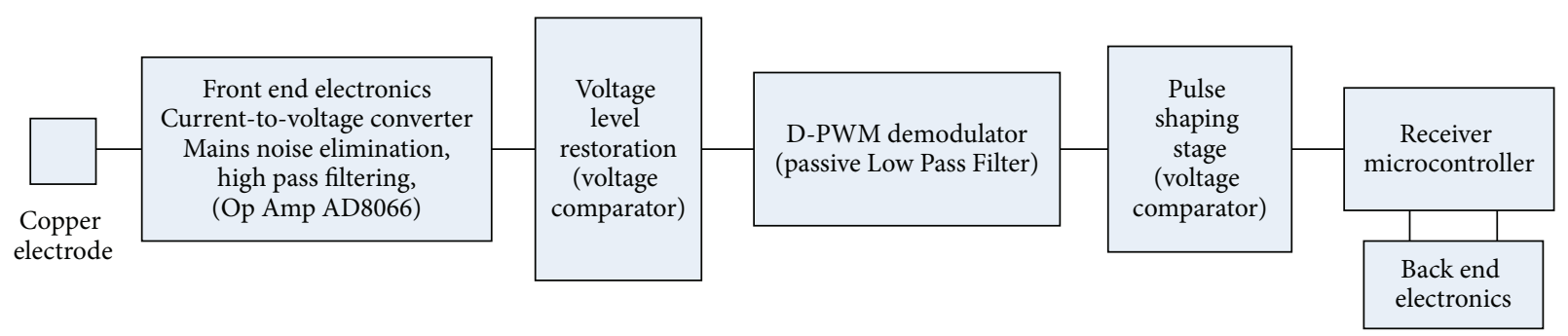

FIGURE 4: Stages (block diagram) of the receiver.

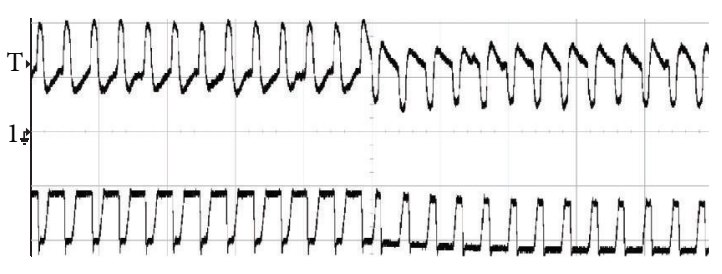

Figure 5: Upper trace: oscilloscope capture at the output of the first (current to voltage converter) stage. It is shown that the low frequency content is lost and the waveform is weighted above and below the zero mean value. Lower trace: output of the second (inverting voltage comparator) stage. The waveform is restored in to its original (two) levels.

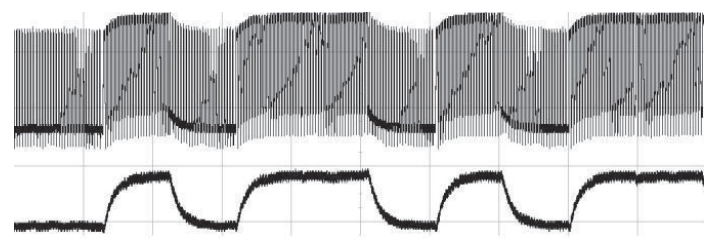

FIgURE 6: Oscilloscope capture at the outputs of the second (voltage comparator, different time scale) as the above and the third (demodulation Low Pass Filter) stages of the receiver. The two different duty cycles give two distinct voltage levels (despite being rounded) corresponding to the logic states.

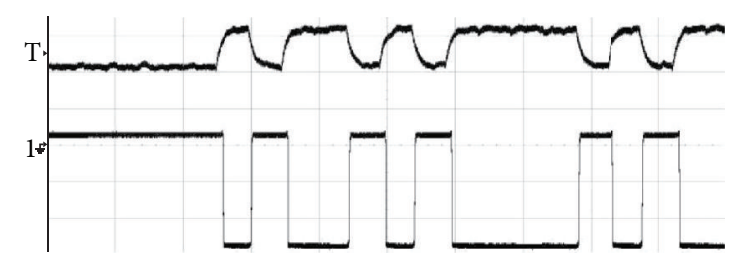

FIgURE 7: Oscilloscope capture at the outputs of the third stage (demodulation Low Pass Filter) as in lower trace of Figure 6 and Pulse Shaping Stage (data output) of the receiver. The signal is finally restored to the voltage levels of the demodulated data waveform.

modulation levels, that is, using more than two symbols (duty cycles) giving the opportunity to pack more bits per symbol in cases where the demand of higher bit rate is necessary. The demodulator, at the receiver side, is also easily implemented as a Low Pass Filter. In most of the cases, there is no need of clock extraction.

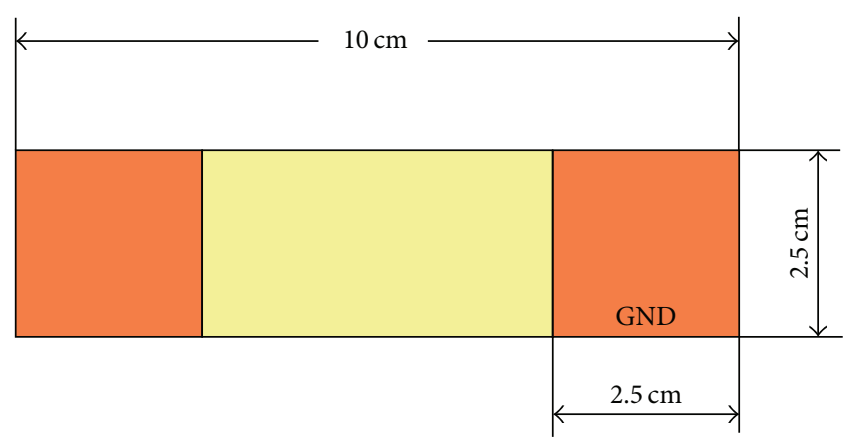

FIGURE 8: Transmitter's coupler electrode. The actual electrode is made from copper covered printed circuit board. The copper has been removed from the middle area.

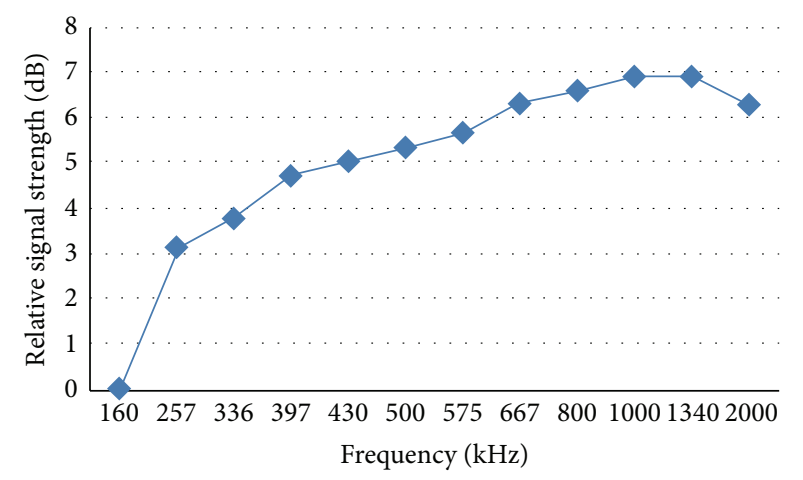

FIGURE 9: Relative signal strength versus frequency.

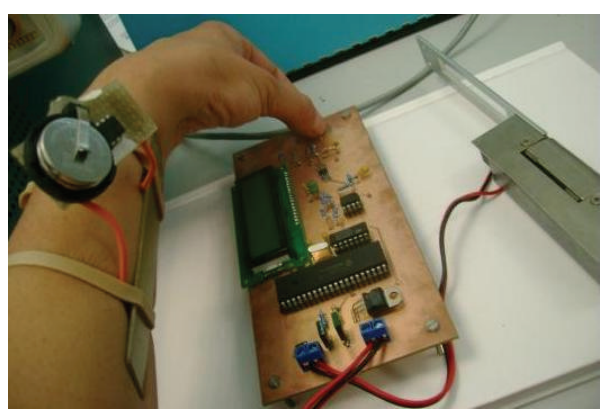

FIgURE 10: Human hand wearing the transmitter and touching the receiver board. At the right side, for demonstration purposes, is shown an electrically operated door lock. 


\section{Conflict of Interests}

The digital modulation method described in this paper is a part of a proposal submitted for possible financing. The decision regarding this financing is pending. In the case of favorable decision, the relevant royalties will be divided among the authors, the finance authority, and the Democritus University of Thrace.

\section{References}

[1] T. G. Zimmerman, "Personal area networks: near-field intrabody communication," IBM Systems Journal, vol. 35, no. 3-4, pp. 609-617, 1996.

[2] N. Seyed Mazloum, Body-coupled communications-experimental characterization, channel modeling and physical layer design [Ph.D. thesis], Chalmers University of Technology, 2008.

[3] M. Wegmueller, A. Lehner, J. Froehlicr et al., "Measurement system for the characterization of the human body as a communication channel at low frequency," in Proceedings of the 27th Annual International Conference of the Engineering in Medicine and Biology Society (IEEE-EMBS '05), pp. 3502-3505, IEEE, September 2005.

[4] G. S. Anderson and C. G. Sodini, "Body coupled communication: the channel and implantable sensors," in Proceedings of the IEEE International Conference on Body Sensor Networks (BSN '13), pp. 1-5, IEEE, Cambridge, Mass, USA, May 2013.

[5] B. Kibret, M. Seyedi, D. T. H. Lai, and M. Faulkner, "The effect of tissues in galvanic coupling intrabody communication," in Proceedings of the IEEE 8th International Conference on Intelligent Sensors, Sensor Networks and Information Processing: Sensing the Future (ISSNIP '13), pp. 318-323, April 2013.

[6] T. Leng, Z. Nie, W. Wang, F. Guan, and L. Wang, "A human body communication transceiver based on on-off keying modulation," in Proceedings of the 2nd International Symposium on Bioelectronics and Bioinformatics (ISBB '11), pp. 61-64, November 2011.

[7] K. Hachisuka, A. Nakata, T. Takeda et al., "Development of wearable intra-body communication devices," Sensors and Actuators A: Physical, vol. 105, no. 1, pp. 109-115, 2003.

[8] K. Partridge, B. Dahlquist, A. Veiseh et al., "Empirical measurements of intrabody communication performance under varied physical configurations," in Proceedings of the 14th Annual ACM Symposium on User Interface Software and Technology (UIST '01), pp. 183-190, ACM, November 2001.

[9] J. A. Ruiz, J. Xu, and S. Shimamoto, "Propagation characteristics of intra-body communications for body area networks," in Proceedings of the 3rd IEEE Consumer Communications and Networking Conference (CCNC '06), vol. 1, pp. 509-513, January 2006.

[10] B. H. E. A. Camelia Gabriel, "European Council recommendation, human exposure to electromagnetic fields high frequency (10 khz to $300 \mathrm{ghz}$ )," in Electricity and Magnetism in Biology and Medicine, pp. 73-76, 1999.

[11] J. Cleveland and F. Robert, "Federal communications commission (FCC), guidelines for human exposure to radiofrequency radiation,radiation, federal register 38653100 (1985)," in Mobile Communications Safety, Telecommunications Technology \& Applications Series, pp. 79-144, Springer US, 1997.
[12] S. D. Kim, S. M. Lee, and S. E. Lee, "Secure communication system for wearable devices wireless intra body communication," in Proceedings of the IEEE International Conference on Consumer Electronics (ICCE '15), pp. 381-382, January 2015.

[13] M. Shinagawa, M. Fukumoto, K. Ochiai, and H. Kyuragi, "A near-field-sensing transceiver for intrabody communication based on the electrooptic effect," IEEE Transactions on Instrumentation and Measurement, vol. 53, no. 6, pp. 1533-1538, 2004.

[14] P. Harikumar, M. I. Kazim, and J. J. Wikner, "An analog receiver front-end for capacitive body-coupled communication," in Proceedings of the NORCHIP Conference, pp. 1-4, IEEE, Cpenhagen, Denmark, November 2012.

[15] M. H. Maruf, A. Korishe, and S. Roy, "Analysis of analog receiver front end sections for body-coupled communication," in Proceedings of the International Conference on Informatics, Electronics and Vision (ICIEV '14), pp. 1-5, IEEE, May 2014.

[16] F. Koshiji, K. Sasaki, D. Muramatsu, and K. Koshiji, "Input impedance characteristics of wearable transmitter electrodes for intra-body communication," in Proceedings of the 1st IEEE Global Conference on Consumer Electronics (GCCE '12), pp. 362363, October 2012.

[17] J. Bae and H.-J. Yoo, "The effects of electrode configuration on body channel communication based on analysis of vertical and horizontal electric dipoles," IEEE Transactions on Microwave Theory and Techniques, vol. 63, no. 4, pp. 1409-1420, 2015.

[18] M. Wang, Z. Wang, J. Li, and F. Wan, "Architectural hardware design of modulator and demodulator for galvanic coupling intra-body communication," in Proceedings of the 7th Biomedical Engineering International Conference (BMEiCON '14), pp. 1-4, November 2014. 

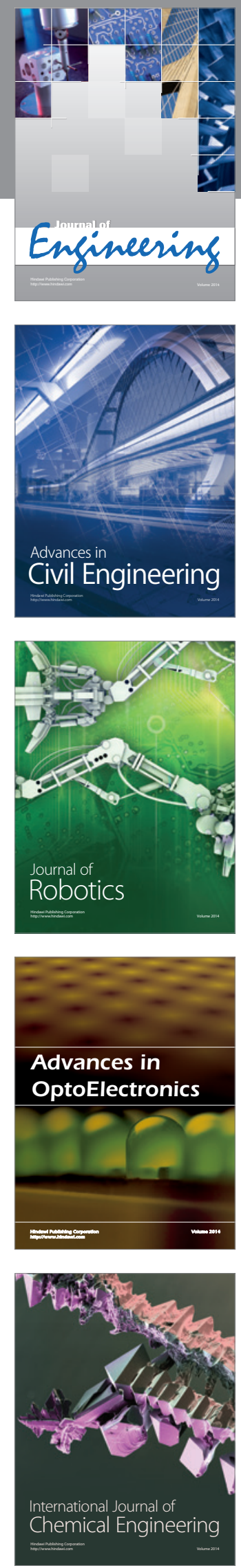

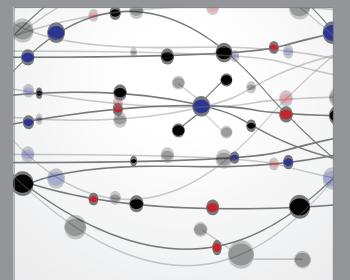

The Scientific World Journal
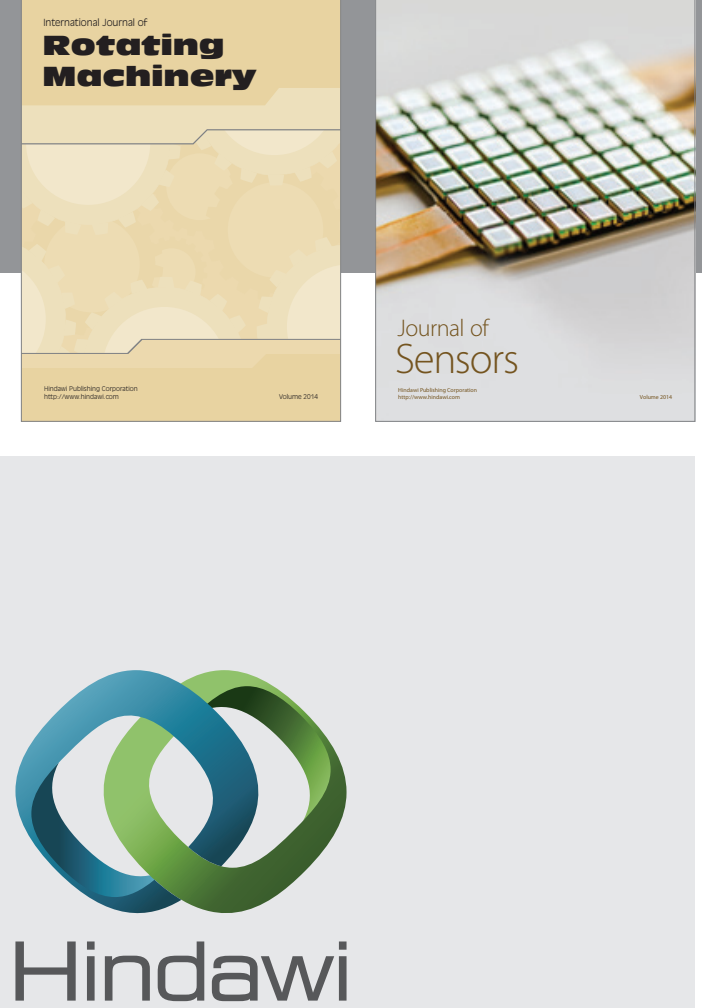

Submit your manuscripts at http://www.hindawi.com
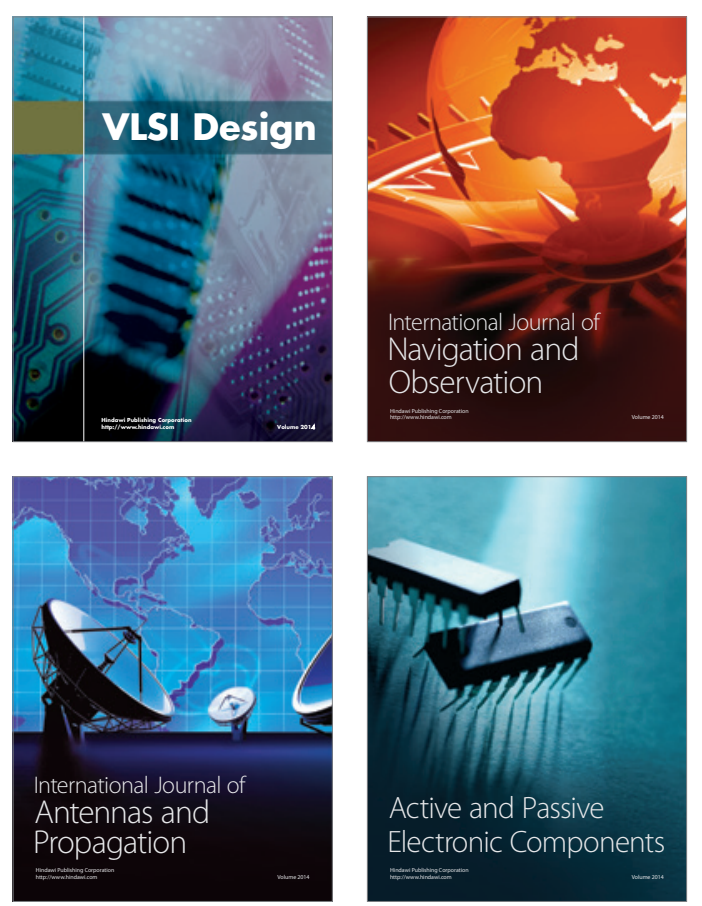
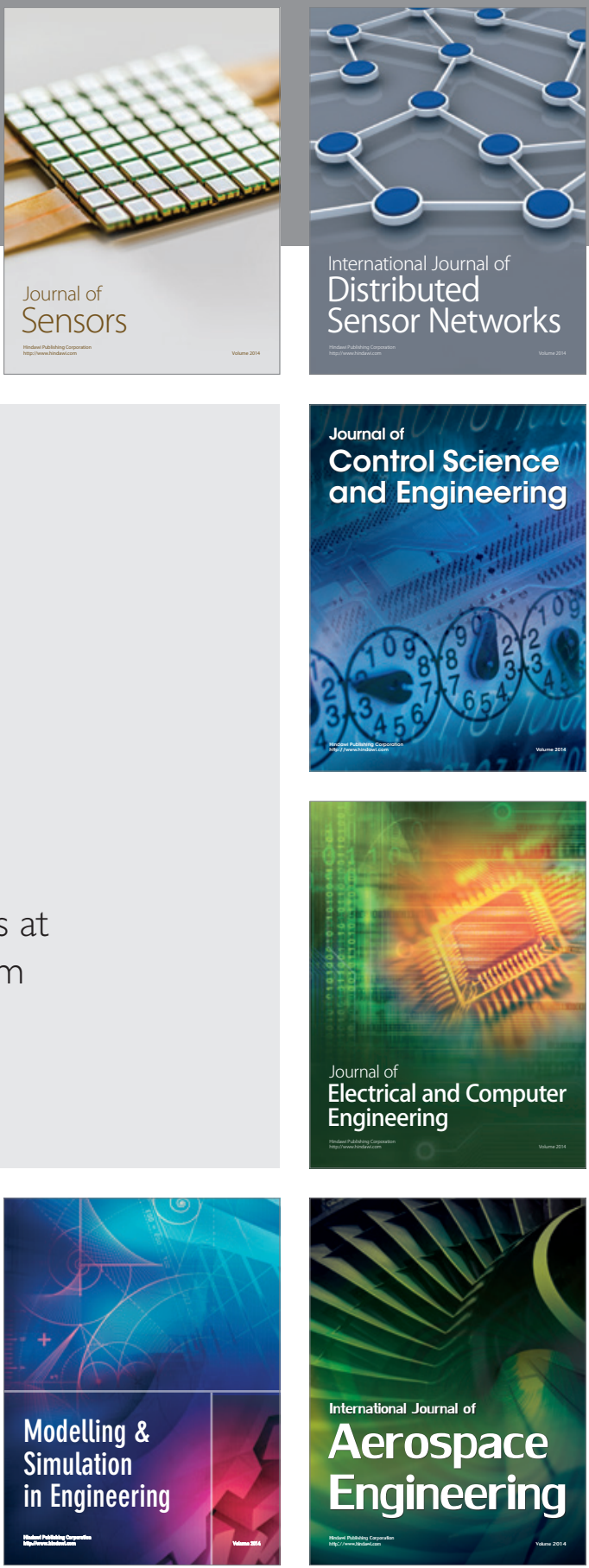

Journal of

Control Science

and Engineering
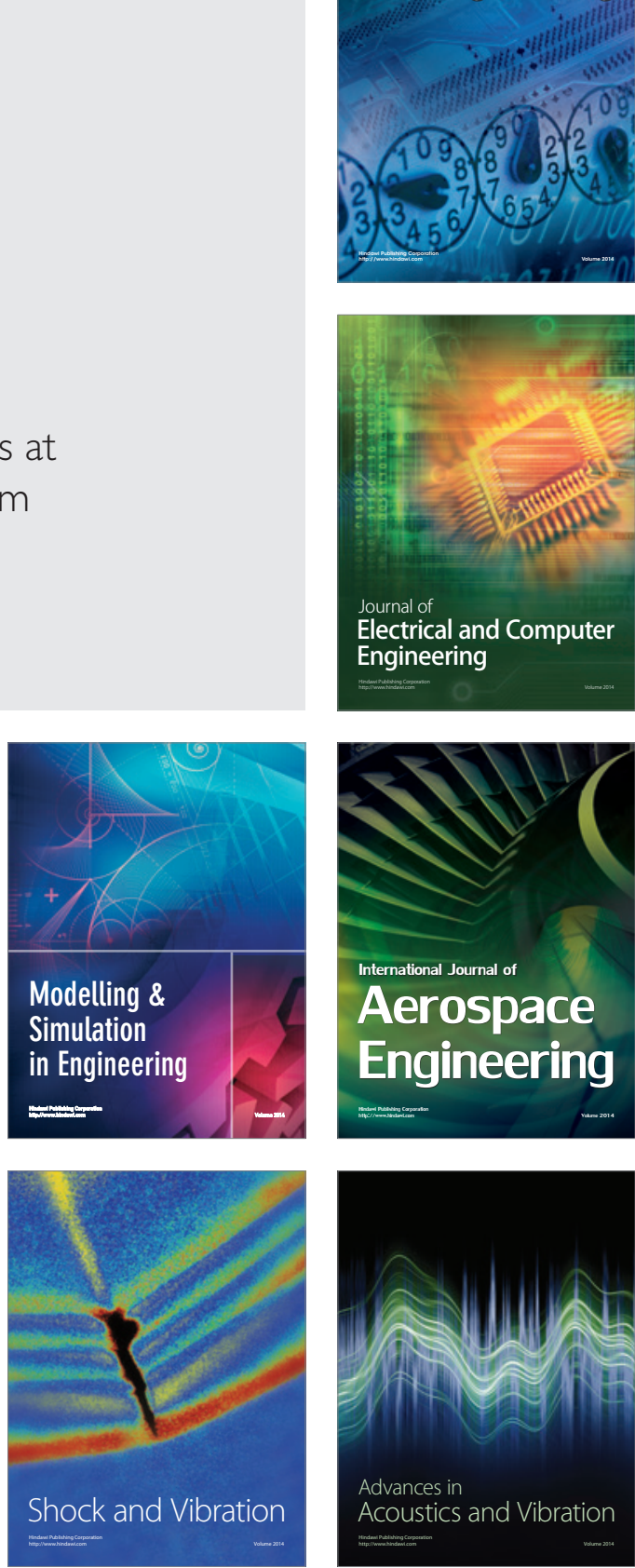